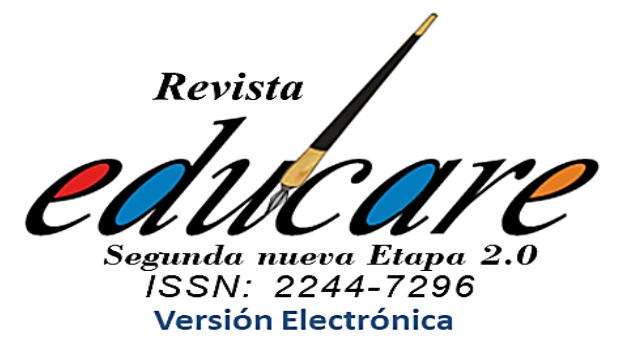

Volumen 25 No. 2 Mayo - Agosto 2021

(401-415)

Ortega Freire, Yanet M.*

ORCID: https://orcid.org/0000-0001-7328-0291

Salazar Cueva, Mónica A.**

ORCID: https://orcid.org/0000-0001-6636-5932

Cayo Lema, Luis E.***

ORCID: https://orcid.org/0000-0001-8947-3135

Pico Macías, Malena E.****

ORCID:https://orcid.org/0000-0003-4221-208X

Universidad Técnica de Cotopaxi

Ecuador

Universidad de las Fuerzas Armadas-ESPE

Ecuador

Universidad Técnica de Manabí

Ecuador

\section{RESILIENCIA Y NEUROCIENCIA. REFLEXIONES EN EL CONTEXTO EDUCATIVO}

Email: Docente (Ciencias Económicas Administrativas y del Comercio) ymortega@espe.edu.ec

* Email: Master (Ciencias Administrativas y de Comercio) masalazar27@espe.edu.ec

**** Email: luis.cayo@utc.edu.ec

**** Email: malena.pico@utm.edu.ec

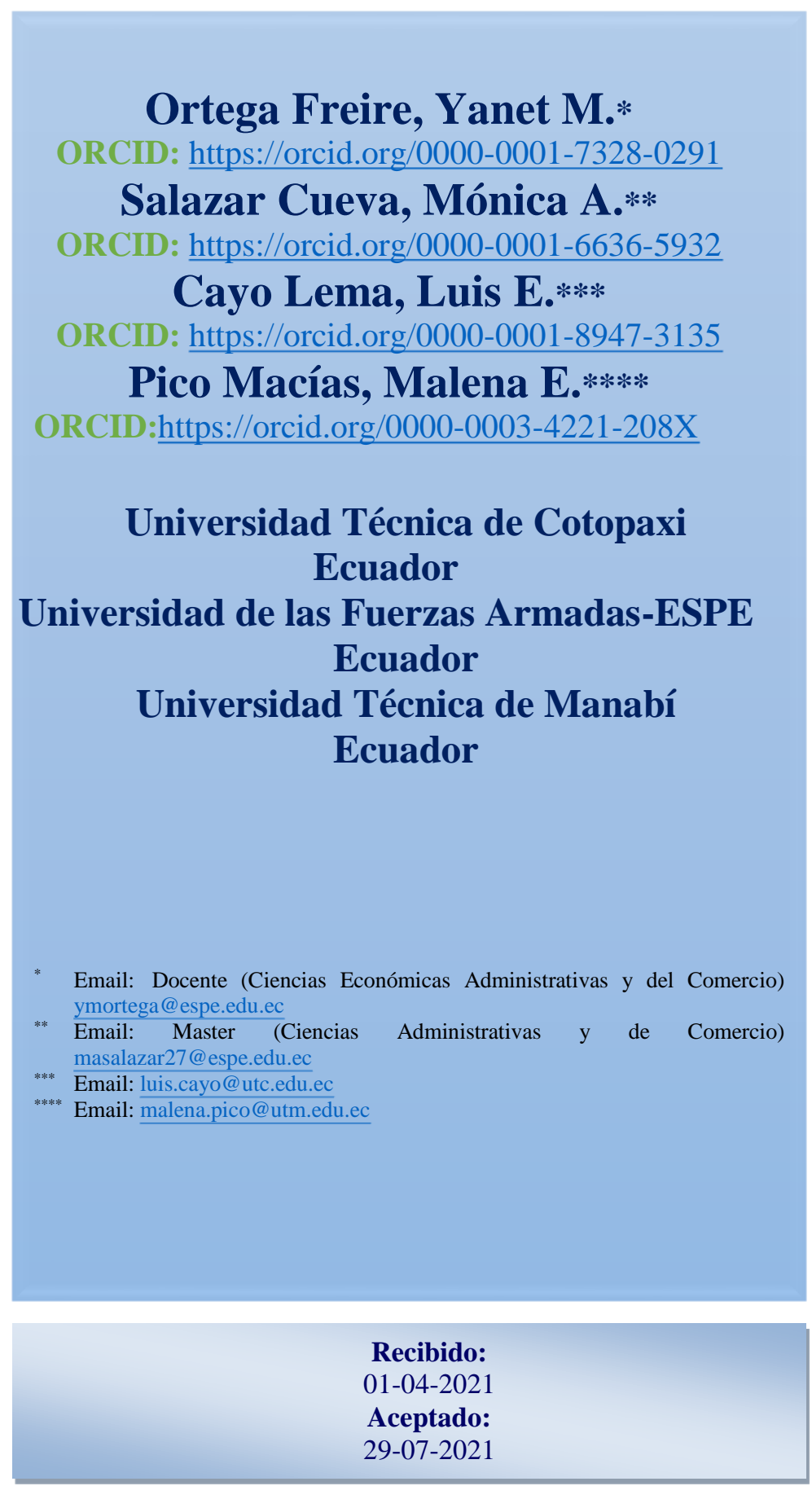




\section{Introducción}

A lo largo de nuestras vidas enfrentamos momentos y situaciones adversas, pero no todas las personas responden de igual manera, entonces ¿cómo funciona nuestra mente ante momentos traumáticos, ante situaciones complicadas? Para indagar sobre este aspecto, la neurociencia ofrece detalles sobre el funcionamiento del cerebro, porque mientras existen personas que se paralizan o toman decisiones nada favorables, existen otras que a pesar de tener todas las condiciones en su contra salen fortalecidos, es decir, estas personas son resilientes.

El término Resiliencia describe la habilidad del ser humano para surgir luego de eventos adversos, lograr adaptarse, recuperarse y poder acceder a una vida significativa y productiva, es una palabra simple, pero encierra un constructo conceptual de cierta complejidad y en la educación se asume como la capacidad del estudiante de sobreponerse con éxito a las dificultades en el aprendizaje. La realidad educativa tiene un gran apoyo en la neurociencia y es a través de la resiliencia que le damos un sí a la vida; por lo tanto, la educación debe sacar la mejor versión del estudiante, los cuales deben motivarse por escenarios innovadores focalizando a la educación hacia la flexibilidad cognitiva, imaginación y creatividad (Gómez, 2010; Ospina y Sarmiento, 2010).

El presente ensayo tiene el propósito de disertar las relaciones entre la Resiliencia y la Neurociencia a partir de sus implicaciones en el contexto educativo. Para ello se procuró realizar el análisis crítico sobre algunas fuentes documentales impresas y electrónicas sobre la temática, que permitiese esbozar las relaciones entre resiliencia y neurociencia, y presentar las posibilidades de esta vinculación en los escenarios educativos, especialmente orientado a la formación docente.

\section{Disertación}

La pandemia de enfermedad por coronavirus (COVID-19) ha provocado una crisis sin precedentes en todos los ámbitos de la sociedad, razón por la cual la educación presenta un gran desafío frente a esta realidad, resulta necesario que las escuelas desplieguen todo su potencial y recursos para fomentar y fortalecer la capacidad interna de los docentes y estudiantes, esto implicaría apoyarse en los avances de las neurociencias para elaborar estrategias que permitan asumir y enfrentar de manera positiva los cambios que se están generando. 
Aunado a esto, vivimos tiempos de cambio conflictivo a múltiples niveles, que afectan a diversos órdenes (académico, social, psicológico, económico y cultural), la época que estamos transitando, conocida como la postmodernidad se caracteriza por prevalecer el individualismo, el hedonismo, relativismo, la permisividad, el consumismo, vale decir, todo un modelo filosófico que impacta el pensamiento y los sentimientos, se inspira en las tecnologías de información y comunicación, propiciando una crisis de la educación contemporánea en la cultura globalizada y una nueva concepción del hombre.

Todo esto nos conduce inevitablemente a uno de los mayores desafíos que enfrenta el conocimiento humano actual, como lo es comprender y tratar de explicar las bases biológicas del aprendizaje y la emoción (neurociencias), es decir, la manera como aprendemos, sentimos, percibimos, y actuamos ante circunstancias adversas (resiliencia).

En concordancia con esta idea, la temática de las neurociencias y la resiliencia representan una prioridad para el sector educativo; entendiéndose el primer término como la ciencia encargada del estudio del cerebro; mientras que la resiliencia como la capacidad humana para enfrentar las adversidades de la vida, superarlas e incluso salir de ellas fortalecidos y transformados positivamente, siendo el contexto educativo el ambiente que le puede ofrecer a sus integrantes las competencias necesarias para confrontar los contratiempos, (Uriarte, 2010).

Si bien es cierto, que hoy día se viven tiempos difíciles y existen realidades como la pobreza, inseguridad, violencia, ausencia de padres, maltratos, carencias afectivas, entre otros, también es cierto que la resiliencia no hará que los problemas desaparezcan, pero permite ampliar la visión del problema y manejarlo de manera adecuada, es decir, se reconoce la existencia de las dificultades, pero se le da mayor fuerza a las competencias y capacidades que poseen las personas.

Por ello, la escuela es el lugar donde se debe construir, desarrollar y generar la resiliencia. Al respecto Barudy y Dantagnan (2005) consideran la afectividad y el vínculo, la toma de conciencia de la realidad, la creatividad, el humor, las expectativas elevadas y la construcción de una historia como factores claves para un clima escolar generador de resiliencia.

Con esos factores, los autores quieren resaltar la importancia que tiene el amor y la afectividad en la infancia para generar resiliencia, de igual manera destacan que a pesar de una realidad desfavorable, hay que demostrarles a los estudiantes las potencialidades que poseen. En lo que respecta al ambiente escolar, éste se debe caracterizar por la alegría, debe ser un contexto agradable, ameno; en relación a los docentes, éstos deben mantener unas expectativas elevadas e 
incentivar a sus estudiantes a esforzarse para superar las adversidades y reflexionar sobre sus historias de vida, esto permitirá compartirlas, comprenderlas, producir empatía en el grupo y fortalecerlos.

Ahora bien, ante este panorama la escuela debe dar respuestas, para formar un hombre integral, con competencias y valores, que le permita superarse, de allí que la escuela necesita transformarse en un escenario en el que la resiliencia actúe como un factor de apoyo y de sostenibilidad para afrontar los constantes retos a los que viven sometidos nuestros centros educativos, tal como lo sostiene Kugelmass (2003).

En concordancia con lo expresado, podemos destacar que en el proceso educativo la resiliencia es importante, pues después de la familia, la escuela constituye un ambiente fundamental para que los estudiantes adquieran las competencias necesarias para salir adelante gracias a su capacidad para sobreponerse a la adversidad. La escuela es una institución que no sólo enseña, sino aprende y necesita aprender de la propia experiencia para poder crecer, mejorar y transformar psicológica y pedagógicamente tanto al alumnado como al profesorado.

Es por ello, que Pérez (2007) sostiene, que aquellos alumnos que han generado un comportamiento resiliente en la escuela, según estudios previos llevados a cabo, han logrado sobreponerse a esas experiencias negativas fortaleciéndose en el proceso, además de haber contado con alguna persona de la familia, de la comunidad o de la escuela, con quien lograron establecer un vínculo positivo. Por tanto, el papel que juega la escuela y, en particular, el docente adquiere todo su valor y revela su complejidad.

La escuela es un escenario de intercambio de aprendizajes, un ente capaz de fomentar una educación integral, holística, sistémica, una institución que tiene la corresponsabilidad junto a la familia y los adultos significativos de constituirse en espacio de formación y socialización, de negociación de conflictos, de fortalecimiento del carácter, un entorno donde se produce una multiplicidad de aprendizajes, entre ellos solventar los problemas, enfrentar las adversidades.

Estos planteamientos permiten vislumbrar que dentro de las ciencias sociales y humanas cobra un significado especial la resiliencia, la cual describe la habilidad del ser humano para surgir luego de eventos adversos, lograr adaptarse, recuperarse y poder acceder a una vida significativa y productiva. Así lo afirma Grotberg (1995), al señalar que "la resiliencia es la capacidad humana para enfrentar, sobreponerse y ser fortalecido o transformado por experiencias de adversidad" (p.20). Resiliencia es una palabra simple, pero encierra un constructo conceptual de cierta 
complejidad que parte de la Psicología, pero ha irrumpido en diferentes ámbitos como en la Sociología, las Ciencias Políticas, Ciencias de la Salud y en la Educación.

En líneas generales, la resiliencia es aplicable a niños, adolescentes y personas adultas, los cuales como individuos resilientes, tienen la capacidad de sobrevivir y superar adversidades, de sobreponerse al caos, de vivir ese proceso el cual lleva implícito dolor y sufrimiento; porque no sólo involucra resistir la desgracia o infortunio que viven, sino que además conlleva a no dejarse abatir, a salir airoso de esa situación y continuar sin traumas, logrando un poder de adaptabilidad que genera al ser humano una fortaleza emocional para paliar cualquier situación que se presente en su vida (Uriarte, 2005).

Dentro de este marco, el término resiliencia ha sido motivo de discusión de varios investigadores, algunos promueven que es algo inherente al ser humano, que está escrito en su código genético y emerge cuando las circunstancias de la vida lo exijan, otros afirman que la resiliencia puede incorporarse a un programa educativo de forma tal, que cualquier persona que asista a la escuela pueda desarrollarlo. Ahora bien, sea cualidad innata o desarrollada, será la que marque la diferencia en el éxito alcanzado dentro del ámbito escolar, familiar y social.

Sobre este particular, las ciencias de la salud han incorporado el término resiliencia en su tarea de realizar una descripción de una serie de procesos complejos que intervienen en la puesta en funcionamiento de los mecanismos biológicos responsables del comportamiento de un individuo, proceso dinámico de desarrollo que permite que éste se adapte positivamente ante situaciones traumáticas y/o estresantes.

Vinculado al concepto de resiliencia, la neurociencia, en su afán de estudiar el sistema nervioso, presenta al cerebro como el órgano ejecutor central del sistema biológico responsable de la misma, porque regula los mecanismos neurobiológicos, cognitivos y psicológicos del individuo vinculados con la respuesta al estrés y la resiliencia (D’Alessio, sf).

Lo anteriormente expuesto, permite señalar que la resiliencia es una herramienta que cada vez cobra mayor importancia en la educación, y quizás estemos en presencia de una nueva pedagogía, debido a que los docentes deben ayudar a desarrollar la personalidad de los estudiantes para que sean capaces de superar acontecimientos adversos a los que se enfrentarán durante la vida, lo cual muestra la relación innegable entre cerebro y educación que nos conlleva a afirmar que se puede trabajar la resiliencia a través de la neurociencia (Segovia-Quesada et al. 2020).

Por otro lado, es prioritario tener claro, que estamos en una época donde la educación 
tradicional no resulta atractiva para los estudiantes, quienes están motivados por escenarios innovadores y eso genera una incertidumbre, la educación debe focalizarse hacia la flexibilidad cognitiva, imaginación y creatividad. Para ello, un escenario ideal sería lograr que, en la formación de docentes, psicopedagogos y todos aquellos profesionales que tengan una relación directa con la educación y los aprendizajes, se incorporaran conocimientos de las funciones cerebrales, de la cual es responsable la neurociencia.

En este sentido, la neurociencia nos explica que el cerebro sigue desarrollándose en la educación primaria, secundaria y universitaria; por ende, es adaptable y necesita ser moldeado y formado. Cuando hay estímulos que resulten de interés para el cerebro, se refuerzan o aparecen nuevas conexiones y eso se conserva a lo largo de la existencia. El aprendizaje comprende cambios y conexiones; el éxito de la enseñanza afecta directamente las funciones del cerebro, modificando y variando las conexiones. Es aquí, donde la neurociencia señala que la plasticidad del cerebro habilita aprendizajes a lo largo de la vida. Se hace necesario resaltar, que la plasticidad cerebral también conocida como plasticidad neuronal se refiere a la formación de aprendizajes y adaptaciones al entorno a través de modificaciones en las estructuras de las redes neuronales (Alcover y Rodríguez, 2012).

Las afirmaciones anteriores sugieren, que la plasticidad cerebral permite producir nuevas neuronas; el aprendizaje es un proceso de desarrollo y es justamente la plasticidad del cerebro la que permite la maleabilidad de éste por la experiencia, esto significa que su estructura y conectividad pueden cambiar con la experiencia. Las neuronas, a lo largo de la vida, continúan siendo capaces de hacer y reforzar nuevas conexiones, pero los cambios más drásticos ocurren durante la infancia y la adolescencia. Por ello, el docente debe entender que, para potenciar la formación integral en sus estudiantes, se deben tomar en consideración para generar modificaciones en el aprendizaje la ejercitación constante, la práctica, el esfuerzo, las horas que han dedicado a sus estudios, las experiencias vividas en la cotidianidad de sus actividades académicas, siendo esto, lo que genera la capacidad que tiene la estructura cerebral del educando para generar el aprendizaje (Codina, 2014).

Es por esta razón, que el docente tiene la necesidad apremiante de formarse en el manejo de contenidos como inteligencias múltiples, sistemas de memoria, sistemas atencionales, entre otros, para lograr imprimir mayor creatividad en la educación, porque el tiempo transcurre y obliga cambios en el referido sistema. En concordancia con lo ya expresado, la neurociencia ha permitido 
saber cómo funciona el cerebro cuando se está estudiando, cuando estamos aprendiendo algo nuevo, porque nacemos con millones de neuronas, nacemos con un gran potencial, percepción, comprensión, atención, memoria y estas capacidades básicas hay que desarrollarlas en competencias.

El entrenamiento neuronal es fundamental, hoy se conoce que el cerebro nunca deja de aprender, a cualquier edad podemos aprender, en la medida que se deje de aprender el cerebro se va limitando. Se aprecia de esta manera, que la neurociencia ha permitido a la educación apropiarse de sus herramientas, para aplicarlas en los espacios educativos, razón por la cual se considera prioritario en la formación del docente cursos relacionados con las estrategias que ofrece esta ciencia, para así comprender mejor el funcionamiento del cerebro y poder así innovar y mejorar el proceso enseñanza aprendizaje. Además, es importante tener claro que el cerebro es el encargado de equilibrar y regular los mecanismos neurobiológicos cognitivos y psicológicos vinculados con la respuesta de resiliencia. Para que nuestro cerebro encuentre el equilibrio neuroquímico, es necesario gestionar de forma adecuada nuestras emociones.

En este punto, se plantea la interrogante ¿qué debe conocer el docente? Es importante que los docentes tengan conocimientos en pedagogía, pues en la praxis pedagógica ellos desarrollan un entramado de acciones, que conjuntamente con los estudiantes, producen la transformación de saberes, valores y actividades. Esta pedagogía incluye: principios y conceptos teóricos de la educación, conocimientos del contenido disciplinar, principios metodológicos para la enseñanza, relación realidad - aula, entre otros; pero hemos fallado en incluir estudiar biológicamente al cerebro y sus funciones. Morín (1999) expresa:

El hombre sólo se completa como ser plenamente humano por y en la cultura. No hay cultura sin cerebro humano (aparato biológico dotado de habilidades para actuar, percibir, saber, aprender), y no hay mente (mind), es decir capacidad de conciencia y pensamiento sin cultura, (p.20).

Si se preguntará a los programas de formación docente, acerca de la alianza cultura-cerebro a la cual Morín (1999) hace referencia, con toda seguridad la mayoría responderían que en el proceso de formación pedagógica de nuestros docentes no se incluye el estudio del cerebro, para comprender las capacidades del ser humano en la construcción del conocimiento.

Es oportuno reflexionar acerca de ¿por qué considerar el estudio de las funciones cerebrales en los procesos educativos? la respuesta pareciera obvia, luego de todas las consideraciones expuestas en párrafos anteriores sobre el aporte de la neurociencia al aprendizaje, la resiliencia y 
la educación. Además, el trabajo pedagógico es humano y esto significa que se debería considerar su integralidad, pues somos parte mental-emocional, social-cultural, somos seres complejos e integrales. La labor pedagógica se fundamenta en la actividad del docente, donde la actividad mental y la cultural interactúan y se acoplan para reconstruir y calificar al mismo ser humano.

Respecto a lo anterior, Flórez (1995) señala que las dimensiones mental y neuronal no se pueden separar, sino que se trata más bien de una unidad dual en constante interacción entre mente y cerebro. Se puede señalar, que el estudio del cerebro proporcionaría a los docentes herramientas conceptuales que les permitirá fundamentar las decisiones que orientan las acciones metodológicas en el contexto educativo.

Esto significaría que, a mayores interconexiones neurales, mayores serán los procesos del pensamiento, por ello los educadores deben tener conocimiento de cómo el estrés, la nutrición, el ejercicio y relajamiento tienen incidencia en el aprendizaje y cómo se ve afectada la actividad cerebral de los estudiantes ante estos factores. Por ejemplo, que el docente conozca que la dieta tiene incidencia en el nivel neuronal, eso significa que influye también en los niveles de atención de los estudiantes; una alimentación adecuada, provista de una cantidad apropiada de proteína, puede significar para el estudiante niveles significativos de atención y en los procesos pedagógicos dicha atención es fundamental.

De la misma manera, otro conocimiento que deben poseer los maestros es que mayormente los neurotransmisores están disponibles durante las primeras horas de la mañana, por lo cual la actividad cerebral tendrá mejores resultados durante este período del día. Esto le proporcionará al docente información acerca de la naturaleza de situaciones de aprendizaje que él promueve según el horario de trabajo. Tal como destaca Salazar (2005), necesariamente se debería tomar en cuenta esta característica del accionar del cerebro para aumentar las posibilidades de éxito de aquellas estrategias didácticas que requieran mayor atención de parte de los estudiantes.

Igualmente, comprender las diferencias significativas entre el hemisferio izquierdo y derecho del cerebro es fundamental en la formación docente, pues ambos conducen el aprendizaje en palabras, matemáticas, música y arte. En una persona saludable los dos hemisferios trabajan interactivamente, pero en algunos casos, las dificultades en el aprendizaje dependen de cuál de los hemisferios esté más desarrollado, por eso las diversas estimulaciones cerebrales pueden producir diferentes resultados.

Por estas razones, en el proceso enseñanza aprendizaje es fundamental el abordaje de 
estrategias didácticas tales como: mapas conceptuales, esquemas mentales, combinación de colores, palabras, imágenes, entre otros, los mismos desarrollarán la actividad cerebral en ambos hemisferios. En otras palabras, la enseñanza es una tarea multifacética que permite a los estudiantes expresarse en forma diferente: visual, táctil, emocional, entre otros; los estilos de aprendizaje tienen su base también en la configuración neuronal de cada persona, porque el cerebro aprende con información con sentido, qué es importante para quien aprende.

Otra consideración importante a tener en cuenta es que los seres humanos somos unidades bio-sociales; de aquí se deriva que las emociones son decisivas en los procesos de aprendizaje. Por ello, lo que aprendemos está influenciado y organizado por emociones y estructuras mentales basadas en expectativas, inclinaciones y prejuicios personales, grados de autoestima y la necesidad de interactuar socialmente. Y es que las emociones son críticas para la elaboración de pautas, lo que aprendemos está organizado e influido por las emociones. Por esta razón, la emoción y la cognición están estrechamente relacionadas y el diseño anatómico cerebral es coherente con esta relación.

En consecuencia, los docentes deben estar conscientes que un clima afectivo y emocional positivo en la institución educativa y en la clase es altamente significativo y debe emplear estrategias efectivas de comunicación que le permita a ambos, estudiantes y docentes, la reflexión sobre los procesos metacognitivos que suponen experiencias de éxito, ya que son detonadores de una acción cerebral que enriquece los procesos de aprendizaje. Además, dichas estrategias deben ser diversas, adaptadas a los estudiantes, porque no todos se emocionan con lo mismo y es por ello que la uniformidad curricular tiende a ser un fracaso.

Lo anterior es reafirmado por Mansilla (2020), cuando señala que la emoción es el motor del aprendizaje, y corresponde a los docentes intentar en su clase emocionar a los estudiantes, avivar su atención y curiosidad, dado que sin ellas no hay aprendizaje. Es aconsejable entonces, que el docente haga una pausa en su explicación en clase cada cierto tiempo y emplee acertijos o cuente chistes, anécdotas, historias (éstas activan estructuras subcorticales y se aprende mejor), que ponga en relieve lo emocional y que capte la atención de los estudiantes.

En relación con estas implicaciones, todo lo señalado anteriormente ha de significar, que el docente debe usar estrategias didácticas que le permita a los estudiantes orquestar experiencias de aprendizaje donde todos los aspectos de la operación del cerebro se consideren, para ello los programas de formación deben preparar al docente en los conocimientos que atañen a los aspectos 
biológicos y funciones del cerebro, a entender cómo se relaciona éste con la manera de aprender, lo cual llevado a la integración de la neurociencia y la educación, que ha supuesto un nuevo paradigma en el ámbito educativo para reflexionar, analizar y explicar el comportamiento del cerebro humano como un órgano que aprende.

La neurociencia nos está indicando cómo son los procesos naturales del aprendizaje en el cerebro, en tanto el docente puede apoyarse en ella para desarrollar la resiliencia en sus estudiantes, es importante entender que la educación debe sostenerse en los adelantos de las ciencias y enseñar en función de lo que se sabe hoy, debemos apoyarnos en ese conocimiento, y debemos llevarlo a la práctica, llevarlo al aula, y colocar al estudiante en el centro, la educación tiene esa responsabilidad, en ayudar a que los estudiantes se autorregulen.

Al respecto Cyrulnik (2005), destaca que se debe cambiar la estructura educativa para dar seguridad a los niños en lugar de amenazarlos y presionarlos por notas altas, a partir de entonces habrá más niños resilientes, no se acabarán las adversidades de la vida, pero podrán afrontarlas mejor y no serán prisioneros de las desgracias.

Sobre este particular, el manejo acertado del educador en el ambiente de clases es fundamental para fortalecer la confianza, la autoestima, el afecto, un alumno que siente que tiene la competencia y el apoyo del maestro tendrá seguridad para afrontar situaciones adversas, por ello los encargados de la educación deben reflexionar y meditar sobre su actuación.

En este mismo orden de ideas, Alonso (2018) destaca que la carencia de afecto y apoyo social en la infancia deterioran el desarrollo de áreas neuronales implicadas en mecanismos de superación de las dificultades y esto conlleva ser más vulnerables frente a eventos negativos, pero gracias a la asombrosa plasticidad del cerebro, expone dicho autor, sabemos que es posible aprender recursos para disminuir los efectos producidos por las experiencias negativas y poder así revertir conductas de abandono y pasividad ante los eventos traumáticos vividos.

Por ello se considera que la educación tiene el gran reto de formar personas en resiliencia, capaces de afrontar las dificultades con entereza, de tal manera que sobrevivan con éxito a las situaciones desfavorables. Dicho de otro modo, tenemos la certeza que en el transcurrir de la vida estaremos expuestos a experiencias traumáticas, complejas, a cambios repentinos que nos afectarán, pero sabemos que tenemos un cerebro que tiene la capacidad de adaptarse a estas situaciones y lograr solucionar el problema, buscando nuevas alternativas.

De allí, que educar a los niños y jóvenes para desarrollar la resiliencia significa educarlos 
para un desarrollo psicológico sano, para su bienestar personal a pesar de las dificultades y para logar el éxito en sus proyectos, mostrarles el camino para que se hagan más fuertes, para que superen mejor las adversidades y así resistir a los contratiempos de la vida. Se debe ver las crisis como una gran oportunidad y esa es la visión que debe introducirse a los estudiantes.

Las afirmaciones anteriores sugieren, que la adversidad fortalece y eso es la premisa que se debe trabajar en el aula de clases, todos tenemos la posibilidad de trascender y superar los desafíos de la vida, tener vínculos humanos profundos, ese contacto humano es primordial para generar bienestar, tal como lo dice Manes (2020), el cerebro se fortalece con los vínculos afectivos.

Por tal razón, el establecimiento de vínculos en las etapas escolares se fortalece con el apoyo y cariño de personas cercanas, familiares, docentes, quienes facilitan los estímulos, los espacios, la afectividad, comunicación, la socialización, la protección, los cuidados y las relaciones de amor. De igual manera, se debe cuidar el vínculo emocional con los alumnos de manera que se sientan aceptados, valorados y queridos, lo cual fomentará el respeto con sus semejantes, con el ambiente y los recursos existentes en el mismo, tales como plantas y animales, además mejorará la comunicación, su autoestima, potenciará su confianza, su libertad, su sensibilidad, la tolerancia, la empatía a la vez que se favorece el desarrollo social, cognitivo y afectivo.

Debido a esto, se debe sacar ventaja de cómo funciona el cerebro para aplicarlo en la enseñanza, para promover la resiliencia en cada niño, en cada joven en el salón de clase, incentivarlos para que aprecien el fracaso como un reto de intentarlo de nuevo y buscar nuevas soluciones.

\section{Reflexiones Finales}

Como reflexiones finales, se tienen las consideraciones que plantea la neurociencia acerca del aprendizaje, lo cual constituye un verdadero desafío para los docentes en el aula, porque en su formación no incorporan contenidos acerca de conocer cómo es el cerebro, cómo aprende, cómo procesa, conserva y evoca una información. Si esto cambiara, ellos podrían emplear ese conocimiento en proponer estrategias de enseñanza, que fomente el aprendizaje impartido en su praxis pedagógica y serían verdaderos agentes significativos en la formación y sus estudiantes, agentes activos en el proceso de aprendizaje.

Se deben repensar los contenidos de la educación, éstos no pueden limitarse sólo en desarrollar la razón intelectual (el cerebro neocortical); la neurociencia ha demostrado que el 
cerebro límbico es el cerebro del amor, de la empatía, de todos los valores ligados a la sensibilidad, por ello el gran reto educativo es enriquecer la razón técnico-científica con la razón cordial, sensible, con el afecto, con el vínculo. Y no existe mejor manera para ello que, a través de cursos formales impartidos en las instituciones educativas para desarrollar estas áreas, pues no existen otras maneras de abordar al hombre en sus distintas etapas del desarrollo, que no sea desde la doble perspectiva biológica y psicológica, como un ser capaz de percibir, amar, sufrir, creer, ilusionarse, desilusionarse, caerse y levantarse.

Cada individuo procesa la información correctamente o la sesga de acuerdo con sus valores o creencias, impulsado por su particular neurobiología con variantes motivacionales o emocionales, por su personalidad, por la influencia de sus seres queridos, por la significatividad de sus docentes o del entorno social y escolar que le rodea.

Por no darle la importancia que se merece a la parte emocional, en los contextos escolares, se aprecia la falta de empatía, el irrespeto al otro, la insensibilidad, lo cual se ha traducido en un fenómeno que conocemos como el acoso escolar o bullying. Vale la pena preguntarse, ¿cuántos problemas psicológicos, bajo rendimiento estudiantil, repitiencia, maltratos, suicidios, se han podido prevenir en las escuelas con el solo hecho de trabajar la resiliencia en clase? Por ello, se hace apremiante incluir dentro de las programaciones, el trabajo de las emociones para fortalecer la resiliencia.

Además, un docente comprometido con su profesión podrá desarrollar procesos de resiliencia, constantemente está enriqueciendo los vínculos, busca solución a los conflictos, mantiene siempre una actitud positiva, es afectuoso, respetuoso, ofrece apoyo, mantiene una comunicación clara y constante, en un motivador, se apoya en las potencialidades y fortalezas, logrando así sensibilizar a sus estudiantes potenciando sus emociones y capacidades cognitivas tal como lo plantea la neurociencia.

Finalmente, se debe precisar que el confinamiento generado por la pandemia COVID 19 nos ha dejado distintas enseñanzas y una nueva cosmovisión que nos acercan a retomar valores trastocados por la postmodernidad: la convivencia, la tolerancia, el cuidado al ambiente, el respeto al otro, el afecto, la empatía, lo cual le da un mayor sentido a la resiliencia en el campo educativo. En este sentido los docentes deben trabajar sobre este aspecto, reforzando la parte afectiva emocional, ayudando a reforzar la confianza y seguridad, a reconocer sus potencialidades, a convertirlos en seres críticos, reflexivos, capaces de resolver problemas, conflictos y darse cuenta 
que tienen las competencias para enfrentarse y superar las adversidades.

\section{Referencias}

Alcover, C. Rodríguez F. (2012) Plasticidad cerebral y hábito en William James: Un antecedente para la Neurociencia Social. Psychologia Latina, 3(1), p.1-9. http://dx.doi.org/10.5209/rev_PSLA.2012.v3.n1.38737

Alonso, J. (2018). Lectura y plasticidad neuronal. Neurociencia. Blog de José Ramón Alonso https://jralonso.es/2018/01/10/lectura-y-plasticidad-neuronal/

Barudy J. y Dantagnan, M. (2005). Los buenos tratos a la infancia. Personalidad, apego y resiliencia. Editorial Gedisa

Codina Felip, M. J. (2014). Neuroeducación en virtudes cordiales. Una propuesta a partir de la neuroeducación y la ética discursiva cordial. [Tesis Doctoral]. https://core.ac.uk/download/pdf/71025424.pdf

Cyrulnik, B. (2005). Los Patitos feos. La resiliencia: una infancia infeliz no determina la vida. Barcelona, España: Gidesa.

D’Alessio, L. (s.f.). Mecanismos neurobiológicos de la resiliencia. Información Científica Gador. Editorial Polemos. https://www.gador.com.ar/wp-content/uploads/2015/04/resiliencia.pdf

Flórez, O., (1995). Hacia una pedagogía del conocimiento. Colombia: McGraw Hill.

Gómez Moreno, B. (2010). Resiliencia individual y familiar. https://www.avntf-evntf.com/wpcontent/uploads/2016/12/GomezB.Trab .3BI0910.pdf

Grotberg, E. (1995). A guide to promoting resilience in children: strengthening the human spirit. The Internacional Resilence Proyect. Early Childhood Development: Practice and Reflections, (8) https://bibalex.org/baifa/attachment/documents/115519.pdf

Kugelmass, J. W. (2003). Inclusive Leadership. Leadership for inclusion. London (UK): National College for School Leadership (NCSL).

Manes, F. (2020). Ser humanos. Planeta

Mansilla, E. (2020). Neurociencia y proceso de enseñanza aprendizaje. Madrid. Revista de Innovación Didáctica de Madrid, 44-62. https://es.calameo.com/read/0002328454e4e76cffc7b 
Morín, E. (1999). Los siete saberes necesarios para la educación del futuro. UNESCO.

Ospina Macías, M. I., y Sarmiento Busquets, P. (2010). Promoción de la resiliencia en la primera infancia un reto para el contexto escolar. [Trabajo de grado]. https://repository.javeriana.edu.co/bitstream/handle/10554/6670/tesis 102.pdf?sequence=1

Pérez Ibarra, A. (2007). La resiliencia: una tarea educativa. Revista de la Universidad de la Salle, (44), 87-93. https://ciencia.lasalle.edu.co/ruls/vol2007/iss44/8/

Salazar, S. (2005). El aporte de la neurociencia para la formación docente. Revista Electrónica Actualidades Investigativas en Educación, 5(1), 1409-4703 https://www.redalyc.org/pdf/447/44750102.pdf

Segovia-Quesada, S., Fuster-Guillén, D., y Ocaña-Fernández, Y. (2020). Resiliencia del docente en situaciones de enseñanza y aprendizaje en escuelas rurales de Perú. Revista Electrónica Educare, 24(2). http://dx.doi.org/10.15359/ree.24-2.20

Uriarte Arciniega, J. (2005). La resiliencia. Una nueva perspectiva en psicopatología del desarrollo. Revista de $\quad$ Psicodidáctica, 79.https://www.redalyc.org/pdf/175/17510206.pdf 